\title{
The Design of Fuzzy Controller by Means of Evolutionary Computing and Neurofuzzy Networks
}

\author{
Sung-Kwun $\mathrm{Oh}^{1}$ and Seok-Beom $\mathrm{Roh}^{2}$ \\ ${ }^{1}$ Department of Electrical Engineering, The University of Suwon, San 2-2 Wau-ri, \\ Bongdam-eup, Hwaseong-si, Gyeonggi-do, 445-743, South Korea \\ ohsk@suwon.ac.kr \\ ${ }^{2}$ Department of Electrical Electronic and Information Engineering, Wonkwang University, \\ 344-2, Shinyong-Dong, Iksan, Chon-Buk, 570-749, South Korea
}

\begin{abstract}
In this study, we propose a new design methodology to design fuzzy controllers. This design methodology results from the use of Computational Intelligence (CI), namely genetic algorithms and neurofuzzy networks (NFN). The crux of the design methodology is based on the selection and determination of optimal values of the scaling factors of the fuzzy controllers, which are essential to the entire optimization process. First, the tuning of the scaling factors of the fuzzy controller is carried out, and then the development of a nonlinear mapping for the scaling factors is realized by using GA based NFN.
\end{abstract}

\section{Introduction}

In the conventional design method to build fuzzy controllers, a control expert proposes some linguistic rules and decides upon the type and parameters of the associated membership functions. With an attempt to enhance the quality of the control knowledge conveyed by the expert, genetic algorithms (GAs) have already started playing a pivotal role. One should stress however that evolutionary computing is computationally intensive and this may be a point of concern when dealing with amount of time available to such search. For instance, when controlling a nonlinear plant such as an inverted pendulum of which initial states vary in each case, the search time required by GAs could be prohibitively high. To alleviate this shortcoming, we introduce a nonlinear mapping from the initial states of the system and the corresponding optimal values of the parameters. With anticipation of the nonlinearity residing within such transformation, in its realization we consider GA-based NFN. Bearing this mind, the development process consists of two main phases. First, using genetic optimization we determine optimal parameters of the fuzzy controller for various initial states of the dynamic system. Second, we build up a nonlinear model that captures a relationship between the initial states of the system and the corresponding genetically optimized control parameters.

\section{Design Methodology of Fuzzy Controller}

The block diagram of fuzzy PID controller is shown in Fig. 1. Note that the input variables to the fuzzy controller are transformed by the scaling factors (GE, GD, GH, 


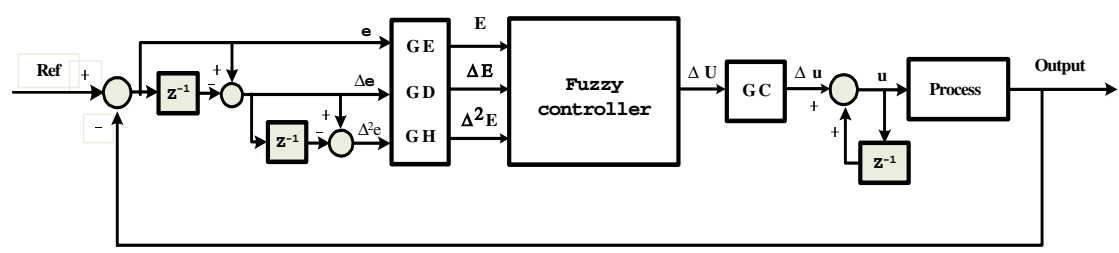

Fig. 1. An overall architecture of the fuzzy PID controller

and GC) whose role is to allow the fuzzy controller to properly "perceive" the external world to be controlled.

The above fuzzy PID controller consists of rules of the following form, cf. [3]

$$
R_{j} \text { : if } E \text { is } A_{1 j} \text { and } \Delta E \text { is } A_{2 j} \text { and } \Delta^{2} E \text { is } A_{3 j} \text { then } \Delta U_{j} \text { is } D_{j}
$$

The capital letters standing in the rule $\left(R_{j}\right)$ denote fuzzy variables whereas $D$ is a numeric value of the control action.

Genetic algorithms (GAs) are the search algorithms inspired by nature in the sense that we exploit a fundamental concept of a survival of the fittest as being encountered in selection mechanisms among species [4]. The genetic search is guided by a reproduction, mutation, and crossover. The standard ITAE expressed for the reference and the output of the system under control is treated as a fitness function [2].

It is the overall design procedure of the fuzzy PID controller realized by means of GAs that consists of the following steps

[step 1] Select the general structure of the fuzzy controller [step 2] Define the number of fuzzy sets and set up initial control rules. [step 3] Form a collection of initial individuals of GAs. [step 4] All the control parameters (GE, GD, GH and GC) are tuned at the same time.

\section{The Estimation Algorithm by Means of GA-Based Neurofuzzy Networks (NFN)}

Let us consider an extension of the network with the fuzzy partition realized by fuzzy relations. Fig. 2 visualizes an architecture of such NFN for two-input and one-output, where each input assumes three membership functions. The circles denote processing units of the NFN. The node indicated $\Pi$ denotes a Cartesian product, whose output is the product of all the incoming signals. And $\mathrm{N}$ denotes the normalization of the membership grades.

The learning of the NFN is realized by adjusting connections of the neurons and as such it follows a standard Back-Propagation (BP) algorithm

$$
w(\text { new })=w(\text { old })+2 \cdot \eta \cdot\left(y_{p}-\hat{y}_{p}\right) \cdot \mu_{i}+\alpha\left(w_{i j}(t)-w_{i j}(t-1)\right)
$$

Where, $\eta$ is a positive learning rate and $\alpha$ is the momentum coefficient. 


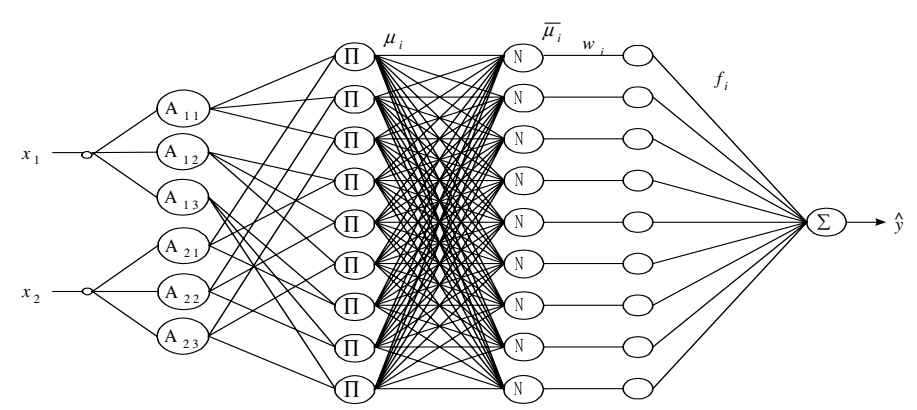

Fig. 2. NFN structure by means of the fuzzy space partition realized by fuzzy relations

\section{Experimental Studies}

In this section, we demonstrate the effectiveness of the fuzzy PID controller by applying it to the inverted pendulum system. Our control goal here is to balance the pole without regard to the cart's position and velocity [7]. We genetically optimize control parameters with a clear intent of achieving the best performance of the controller. GAs are powerful nonlinear optimization techniques. However, the powerful performance is obtained at the cost of expensive computational requirements and much time. To overcome this weak point, we propose the nonlinear model (GA-based NFN) which is able to estimate the control parameters quickly in the case that the initial angular positions and velocities of the inverted pendulum are selected arbitrarily within the given range. Fig. 3 demonstrates (a)pole angle (b)pole angular velocity for initial angle $\theta=0.22(\mathrm{rad})$ and initial angular velocity $\dot{\theta}=0.22(\mathrm{rad} / \mathrm{sec})$.

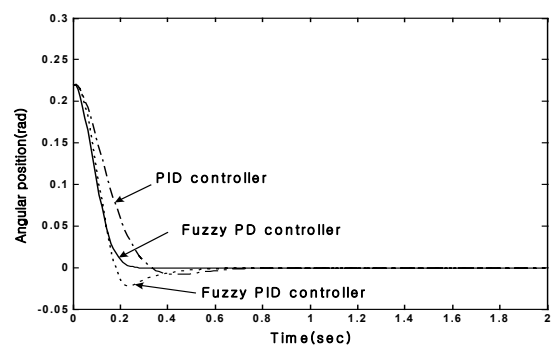

(a)

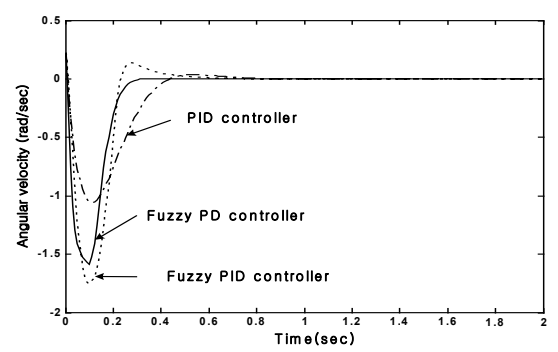

(b)

Fig. 3. (a)pole angle (b)pole angular velocity for initial angle $\theta=0.22(\mathrm{rad})$ and initial angular velocity $\dot{\theta}=0.22(\mathrm{rad} / \mathrm{sec})$

From the above Fig. 3, we know that the fuzzy PD and fuzzy PID control effectively the inverted pendulum system. The output performance of the fuzzy controllers such as the fuzzy PD and the fuzzy PID controller including nonlinear characteristics are superior to that of the PID controller especially when using the nonlinear dynamic equation of the inverted pendulum. Moreover, the proposed estimation algorithm such 
as GA-based NFN generates the preferred model architectures. Especially the fuzzy PD controller describes the preferred one among the controllers.

\section{Conclusions}

We have proposed a two-phase optimization scheme of the fuzzy PID and PD controllers. The first phase of the design of the controller uses genetic computing that aims at the global optimization of its scaling factors where they are optimized with regard to a finite collection of initial conditions of the system under control. In the second phase, we construct a nonlinear mapping between the initial conditions of the system and the corresponding values of the scaling factors. From the simulation studies, using genetic optimization and the estimation algorithm of the GA-based neurofuzzy networks model, we showed that the fuzzy PI/PID controller controls effectively the inverted pendulum system. While the study showed the development of the controller in the experimental framework of control of a specific dynamic system (inverted pendulum), this methodology is general and can be directly utilized to any other system.

Acknowledgement. This work has been supported by KESRI(R-2004-B-274), which is funded by MOCIE(Ministry of commerce, industry and energy)

\section{References}

1. Oh, S.K., Pedrycz, W.: The Design of Hybrid Fuzzy Controllers Based on Genetic Algorithms and Estimation Techniques. Kybernetes 31 (2002) 909-917

2. Oh, S.K., Ahn, T., Hwang, H., Park, J., Woo, K.: Design of a Hybrid Fuzzy Controller with the Optimal Auto-tuning Method. Journal of Control, Automation and Systems Engineering. 1 (1995) 63-70

3. Li, H.X.: A comparative design and tuning for conventional fuzzy control. IEEE Trans. Syst., Man, Cybern. B. 27 (1997) 884-889

4. Goldberg, D.E.: Genetic algorithms in Search, Optimization, and Machine Learning. Addison-Wesley (1989)

5. Yamakawa, T.: A New Effective Learning Algorithm for a Neo Fuzzy Neuron Model. 5th IFSA World Conference. (1993) 1017-1020

6. Park, B.J., Oh, S.K., Pedrycz, W.: The Hybrid Multi-layer Inference Architecture and Algorithm of FPNN Based on FNN and PNN. 9th IFSA World Congress. (2001) 1361-1366.

7. Jang, J.R.: Self-Learning Fuzzy Controllers Based on Temporal Back Propagation. IEEE Trans. On Neural Networks. 3 (1992) 714-723

8. Oh, S.K., Rho, S.B., Kim, H.K.: Fuzzy Controller Design by eans of Genetic Optimization and NFN-Based Estimation Technique. International journal of Control, Automations, and Systems. 2(3) (2004) 362-373 